Bull. Korean Math. Soc. 50 (2013), No. 6, pp. 1765-1780

http://dx.doi.org/10.4134/BKMS.2013.50.6.1765

\title{
SOME TYPES OF REACTION-DIFFUSION SYSTEMS WITH NONLOCAL BOUNDARY CONDITIONS
}

\author{
Yuzhu Han and Wenjie GaO
}

\begin{abstract}
This paper deals with some types of semilinear parabolic systems with localized or nonlocal sources and nonlocal boundary conditions. The authors first derive some global existence and blow-up criteria. And then, for blow-up solutions, they study the global blow-up property as well as the precise blow-up rate estimates, which has been seldom studied until now.
\end{abstract}

\section{Introduction}

In this paper, we investigate several types of reaction-diffusion systems with localized or nonlocal sources and nonlocal boundary conditions. We first study the following two power-type systems with localized reaction terms

$$
u_{t}=\Delta u+v^{p}\left(x_{0}, t\right), \quad v_{t}=\Delta v+u^{q}\left(x_{0}, t\right), x \in \Omega, t>0,
$$

and with nonlocal sources

$$
u_{t}=\Delta u+\int_{\Omega} v^{p}(y, t) \mathrm{d} y, v_{t}=\Delta v+\int_{\Omega} u^{q}(y, t) \mathrm{d} y, x \in \Omega, t>0,
$$

respectively, where $\Omega \subset \mathbb{R}^{N}(N \geq 1)$ is a bounded domain with smooth boundary $\partial \Omega, x_{0}$ is a fixed point in $\Omega$ and $p, q>0$. And then, we investigate two exponent-type systems with localized sources

$$
u_{t}=\Delta u+\lambda e^{p v\left(x_{0}, t\right)}, v_{t}=\Delta v+\mu e^{q u\left(x_{0}, t\right)}, x \in \Omega, t>0,
$$

and with nonlocal sources

$$
u_{t}=\Delta u+\lambda \int_{\Omega} e^{p v(y, t)} \mathrm{d} y, v_{t}=\Delta v+\mu \int_{\Omega} e^{q u(y, t)} \mathrm{d} y, x \in \Omega, t>0,
$$

Received May 10, 2011

2010 Mathematics Subject Classification. 35K51, 35K58.

Key words and phrases. localized source, nonlocal source, nonlocal boundary condition, blow-up profile, blow-up rate.

The project is supported by Key Lab of Symbolic Computation and Knowledge Engineering of Ministry of Education, by the 985 program of Jilin University and by Graduate Innovation Fund of Jilin University (20111034). 
respectively, where $\lambda, \mu, p, q>0$ are constants. Each system will be considered in $\Omega$ with the following nonlocal boundary conditions and initial data

$(1.5)$

$$
\left\{\begin{array}{l}
u(x, t)=\int_{\Omega} \varphi(x, y) u(y, t) \mathrm{d} y, v(x, t)=\int_{\Omega} \psi(x, y) v(y, t) \mathrm{d} y, x \in \partial \Omega, t>0, \\
u(x, 0)=u_{0}(x), \quad v(x, 0)=v_{0}(x), \quad x \in \Omega .
\end{array}\right.
$$

Most physical settings lead to the default assumption that the functions $\varphi(x, y)$, $\psi(x, y)$ defined for $(x, y) \in \partial \Omega \times \bar{\Omega}$ are nonnegative and continuous, and that the initial data $u_{0}(x), v_{0}(x) \in C^{1}(\bar{\Omega})$ are nonnegative. These assumptions are mathematically convenient and currently followed throughout this paper. We also assume that $\left(u_{0}, v_{0}\right)$ satisfies the compatibility condition on $\partial \Omega$, and that $\varphi(x, \cdot) \not \equiv 0$ and $\psi(x, \cdot) \not \equiv 0$ for any $x \in \partial \Omega$ for the sake of the meaning of nonlocal boundary conditions.

Systems (1.1) and (1.3) describe some physical phenomena in which the nonlinear reaction in a dynamical system takes place only at a single point (see $[3,13,17,24])$. Systems (1.2) and (1.4) are related to some ignition models for compressible reactive gases (see $[2,24]$ ).

Over the past few decades, a considerable effort has been devoted to studying the blow-up properties of solutions to parabolic equations with local boundary conditions, say Dirichlet, Neumann, or Robin boundary condition, which can be used to describe heat propagation on the boundary of container (see the survey paper $[12,15])$. In [5], Chadam et al. studied the following single equations with localized source

$$
u_{t}=\Delta u+f\left(u\left(x_{0}, t\right)\right), \quad(x, t) \in \Omega \times(0, T),
$$

and with nonlocal term

$$
u_{t}=\Delta u+\int_{\Omega} f(u(y, t)) \mathrm{d} y, \quad(x, t) \in \Omega \times(0, T),
$$

respectively, with Neumann boundary conditions. They showed some sufficient conditions for the solutions to blow up in finite time, and also proved that the blow-up set in the whole domain $\bar{\Omega}$ whenever a solution blows up in a finite time $T$. In the case of (1.6), they also proved that $u\left(x_{0}, t\right) \leq(2 /(p-1)(T-t))^{1 /(p-1)}$ for $f(s)=s^{p},(p>1)$, and $u\left(x_{0}, t\right) \leq \ln 2 /(T-t)$ for $f(s)=e^{s}$, if the initial datum $u_{0}(x)$ satisfies $\Delta u_{0}(x) \geq 0$. Later, Wang and Wang [27] investigated the heat equation with a nonlocal source and a local absorption term

$$
u_{t}-\Delta u=\int_{\Omega} u^{p}(y, t) \mathrm{d} y-k u^{p}
$$

coupled with homogeneous Dirichlet and Neumann boundary conditions. It was shown that the blow-up occurs for large initial data if $q>p>1$, while all solutions exist globally if $1 \leq q<p$. As for the critical case $p=q$, they proved that whether or not the solutions blow up in finite time depending on the comparison of $|\Omega|$ and $k$. Global blow-up property was also proved. For more 
works on parabolic equations or systems with localized or nonlocal sources, we refer the readers to $[1,6,18,25,29]$.

On the other hand, parabolic equations with nonlocal boundary conditions also arise naturally in applied sciences (see $[4,9,10]$ ). For example, in the study of heat conduction within linear thermoelasticity, Day $[9,10]$ investigated a heat equation coupled with nonlocal boundary condition. Later, Friedman [7] generalized Day's results to a parabolic equation

$$
u_{t}=\Delta u+g(x, u), x \in \Omega, t>0,
$$

which is subject to the following nonlocal boundary condition

$$
u(x, t)=\int_{\Omega} k(x, y) u(y, t) \mathrm{d} y .
$$

He established the global existence of classical solution and discussed its monotonic decay property. Comparison principle and some other further results were also obtained for Problem (1.9) coupled with nonlocal boundary condition (see $[11,22,23,31])$, and some of these results were extended to quasilinear parabolic equations by Wang et al. (see [26]). For other works related to nonlocal boundary conditions, we refer the interested readers to [14, 19, 20, 21, 30, 32]. In particular, by using some ideas of Souplet [24], Kong and Wang [14] obtained the blow-up conditions and blow-up profile of the following system

$$
\begin{cases}u_{t}=\Delta u+\int_{\Omega} u^{m}(x, t) v^{n}(x, t) \mathrm{d} x, & x \in \Omega, t>0, \\ v_{t}=\Delta v+\int_{\Omega} u^{p}(x, t) v^{q}(x, t) \mathrm{d} x, & x \in \Omega, t>0\end{cases}
$$

subject to nonlocal boundary condition (1.5), and Zheng and Kong gave the sufficient conditions for global existence or blow-up of solutions to the following similar system

$$
u_{t}=\Delta u+u^{m} \int_{\Omega} v^{n}(x, t) \mathrm{d} x, v_{t}=\Delta v+v^{q} \int_{\Omega} u^{p}(x, t) \mathrm{d} x, x \in \Omega, t>0
$$

coupled with the same nonlocal boundary condition. The typical characterization of systems (1.11) and (1.12) is the complete couple of the nonlocal sources, which leads to the analysis of simultaneous blow-up.

Motivated by the above works, we intend to study Problems (1.1)-(1.4) with nonlocal boundary conditions. It is our purpose to investigate the roles of weight functions in the blow-up properties of solutions to these systems. It should be remarked that in the works mentioned above, blow-up rates or blowup profile results were derived under the conditions that

$$
\int_{\Omega} \varphi(x, y) \mathrm{d} y, \quad \int_{\Omega} \psi(x, y) \mathrm{d} y \leq 1 \quad \text { for all } x \in \partial \Omega .
$$

In this article, blow-up profiles are studied in the case that

$$
\int_{\Omega} \varphi(x, y) \mathrm{d} y, \quad \int_{\Omega} \psi(x, y) \mathrm{d} y \geq 1 \quad \text { for all } x \in \partial \Omega,
$$

which has been seldom studied until now. 
The rest of this paper is organized as follows. In Section 2, we present some preliminaries including the comparison principle and local existence of a nonnegative solution to Problems (1.1)-(1.4) with condition (1.5). Global existence and blow-up results will be given in Section 3. In Section 4, we investigate the blow-up profile of blow-up solutions of Problems (1.1)-(1.4) for a special case. We shall concentrate our main consideration on Systems (1.1), (1.5) and (1.3), (1.5). For the conclusions of (1.2), (1.5) and (1.4), (1.5), we only give a sketch of the proofs.

\section{Preliminaries}

In this section, we use sub and supersolution technique to prove the global existence and blow-up results. We first remark that local existence of nonnegative classical solutions of (1.1)-(1.4) with (1.5) can be proved by similar arguments as [11]. Moreover, if the maximal existence time $T<+\infty$, then we easily deduce $\lim _{t \rightarrow T}\left(\|u\|_{\infty}+\|v\|_{\infty}\right)=+\infty$. We begin this section with the definition of sub and supersolution of systems (1.1)-(1.4) with condition (1.5). For convenience, we denote $Q_{T}=\Omega \times(0, T)$ and $S_{T}=\partial \Omega \times(0, T)$.

Definition 2.1. A vector valued function $(u, v) \in\left[C^{2,1}\left(Q_{T}\right) \cap C\left(\bar{Q}_{T}\right)\right]^{2}$ is called a subsolution of Problems (1.1), (1.5) if

$$
\begin{cases}u_{t}-\Delta u \leq v^{p}\left(x_{0}, t\right), & (x, t) \in Q_{T}, \\ v_{t}-\Delta v \leq u^{q}\left(x_{0}, t\right), & (x, t) \in Q_{T}, \\ u(x, t) \leq \int_{\Omega} \varphi(x, y) u(y, t) d y, & (x, t) \in S_{T}, \\ v(x, t) \leq \int_{\Omega} \psi(x, y) v(y, t) d y, & (x, t) \in S_{T}, \\ u(x, 0) \leq u_{0}(x), v(x, 0) \leq v_{0}(x), & x \in \Omega .\end{cases}
$$

A supersolution is defined with each inequality reversed. A function is called a solution of Problems (1.1), (1.5) if it is both a subsolution and a supersolution.

Sub and supersolutions of other problems can be defined similarly.

We next give a positivity lemma, which will play important roles in the following discussion.

Lemma 2.1. Suppose that $c_{1}, c_{2}, \varphi$ and $\psi$ are nonnegative functions in their respective domains. If $\omega_{1}, \omega_{2} \in C^{2,1}\left(Q_{T}\right) \cap C\left(\bar{Q}_{T}\right)$ satisfy

$$
\begin{cases}\omega_{1 t}-\Delta \omega_{1} \geq c_{1}(x, t) \omega_{2}\left(x_{0}, t\right), & (x, t) \in Q_{T}, \\ \omega_{2 t}-\Delta \omega_{2} \geq c_{2}(x, t) \omega_{1}\left(x_{0}, t\right), & (x, t) \in Q_{T} \\ \omega_{1}(x, t) \geq \int_{\Omega} \varphi(x, y) \omega_{1}(y, t) d y, & (x, t) \in S_{T} \\ \omega_{2}(x, t) \geq \int_{\Omega} \psi(x, y) \omega_{2}(y, t) d y, & (x, t) \in S_{T} \\ \omega_{1}(x, 0)>0, \omega_{2}(x, 0)>0, & x \in \Omega,\end{cases}
$$

then $\omega_{1}>0, \omega_{2}>0$ on $\bar{Q}_{T}$. 
Proof. The method for proving this lemma is more or less standard. We shall sketch the arguments here for the convenience of the readers.

Set $t_{1}=\sup \left\{t \in(0, T): \omega_{i}(x, t)>0,(i=1,2)\right\}$. Since $\omega_{1}(x, 0), \omega_{2}(x, 0)>$ 0 , by continuity, there exists $\delta>0$ such that $\omega_{1}(x, t), \omega_{2}(x, t)>0$ for all $(x, t) \in \bar{\Omega} \times[0, \delta)$. Thus $t_{1} \in(\delta, T]$.

We shall show that $t_{1}=T$. Assume on the contrary that $t_{1}<T$. Then we have $\omega_{1}\left(x_{1}, t_{1}\right)=0$ or $\omega_{2}\left(x_{1}, t_{1}\right)=0$ for some $x_{1} \in \bar{\Omega}$. Without loss of generality, we may suppose that $\omega_{1}\left(x_{1}, t_{1}\right)=0=\inf _{Q_{t_{1}}} \omega_{1}$.

If $x \in \Omega$, we first notice that

$$
\omega_{1 t}-\Delta \omega_{1} \geq c_{1} \omega_{2}\left(x_{0}, t\right) \geq 0,(x, t) \in \Omega \times\left(0, t_{1}\right] .
$$

In addition, it is clear that $\omega_{1} \geq 0$ for $(x, t) \in \partial \Omega \times\left(0, t_{1}\right) \cup \bar{\Omega} \times\{t=0\}$. Then it follows from the strong maximum principle that $\omega_{1} \equiv 0$ in $Q_{t_{1}}$, which contradicts to $\omega_{1}(x, 0)>0$.

If $x \in \partial \Omega$, we have

$$
0=\omega_{1}\left(x_{1}, t_{1}\right) \geq \int_{\Omega} \varphi(x, y) \omega_{1}\left(y, t_{1}\right) \mathrm{d} y>0,
$$

where we have used the facts that $\varphi(x, \cdot) \not \equiv 0$ for any $x \in \partial \Omega$ and $\omega_{1}\left(y, t_{1}\right)>0$ for any $y \in \Omega$. Again we obtain a contradiction.

Therefore, the claim is true and thus $t_{1}=T$, which implies that $\omega_{1}, \omega_{2}>0$ on $\bar{Q}_{T}$. The proof is complete.

Remark 2.1. If $\int_{\Omega} \varphi(x, y) \mathrm{d} y \leq 1$ and $\int_{\Omega} \psi(x, y) \mathrm{d} y \leq 1$ for all $x \in \partial \Omega$ in Lemma 2.1, we can deduce from $\left(\omega_{1}(x, 0), \omega_{2}(x, 0)\right) \geq(0,0)$ for all $x \in \bar{\Omega}$ that $\left(\omega_{1}(x, t), \omega_{2}(x, t)\right) \geq(0,0)$ on $\bar{Q}_{T}$. In fact, for any $\epsilon>0$, we can conclude that $\left(\omega_{1}(x, t)+\epsilon e^{t}, \omega_{2}(x, t)+\epsilon e^{t}\right)>(0,0)$ on $\bar{Q}_{T}$, by using similar arguments as in the proof of Lemma 2.1. Then the desired result follows from the limit procedure $\epsilon \rightarrow 0$.

Corresponding to Problems (1.2) and (1.4), we have the following lemma which is similar to Lemma 2.1.

Lemma 2.2. Suppose that $c_{1}, c_{2}, \varphi$ and $\psi$ are nonnegative functions in their respective domains. If $\omega_{1}, \omega_{2} \in C^{2,1}\left(Q_{T}\right) \cap C\left(\bar{Q}_{T}\right)$ satisfy

$$
\begin{cases}\omega_{1 t}-\Delta \omega_{1} \geq \int_{\Omega} c_{1}(y, t) \omega_{2}(y, t) \mathrm{d} y, & (x, t) \in Q_{T}, \\ \omega_{2 t}-\Delta \omega_{2} \geq \int_{\Omega} c_{2}(y, t) \omega_{1}(y, t) \mathrm{d} y, & (x, t) \in Q_{T}, \\ \omega_{1}(x, t) \geq \int_{\Omega} \varphi(x, y) \omega_{1}(y, t) d y, & (x, t) \in S_{T}, \\ \omega_{2}(x, t) \geq \int_{\Omega} \psi(x, y) \omega_{2}(y, t) d y, & (x, t) \in S_{T}, \\ \omega_{1}(x, 0)>0, \omega_{2}(x, 0)>0, & x \in \Omega,\end{cases}
$$

then $\omega_{1}>0, \omega_{2}>0$ on $\bar{Q}_{T}$.

Proof. By using similar arguments as in Lemma 2.1, we can prove this lemma. Details are omitted here. 
From the above lemmas, we obtain the following comparison principle by standard arguments.

Proposition 2.1. Let $(\underline{u}, \underline{v})$ and $(\bar{u}, \bar{v})$ be a subsolution and a supersolution of Problem (1.1) (or (1.2), or (1.3), or (1.4)), (1.5) in $Q_{T}$, respectively. If $(\underline{u}(x, 0), \underline{v}(x, 0))<(\bar{u}(x, 0), \bar{v}(x, 0))$ for $x \in \bar{\Omega}$, then $(\underline{u}, \underline{v})<(\bar{u}, \bar{v})$ on $\bar{Q}_{T}$.

\section{Global existence and blow-up}

In this section, we shall use super and subsolution technique to prove the global existence and finite time blow-up results of solutions to our systems. We first introduce a lemma as a preliminary for the proof of global existence results.

Lemma 3.1. Let $f(x, y)$ and $\omega_{0}(x)$ be continuous, nonnegative functions on $\partial \Omega \times \bar{\Omega}$ and $\bar{\Omega}$, respectively, and the nonnegative constants $\theta_{1}, \theta_{2}, \theta_{3}, \theta_{4}$ satisfy $0<\theta_{1}+\theta_{2} \leq 1,0<\theta_{3}+\theta_{4} \leq 1$. Then the solutions of the nonlocal problem

$$
\begin{cases}\omega_{t}-\Delta \omega=\omega^{\theta_{1}} \omega^{\theta_{2}}\left(x_{0}, t\right)+\omega^{\theta_{3}} \omega^{\theta_{4}}\left(x_{0}, t\right), & x \in \Omega, t>0, \\ \omega(x, t)=\int_{\Omega} f(x, y) \omega(y, t) \mathrm{d} y, & x \in \partial \Omega, t>0, \\ \omega(x, 0)=\omega_{0}(x), & x \in \Omega\end{cases}
$$

are global.

Proof. We can easily choose a positive function $\phi_{1}(x) \in C^{2}(\bar{\Omega})$ satisfying

$$
\min _{\bar{\Omega}} \phi_{1}(x)>\max _{\bar{\Omega}} \omega_{0}^{2}(x)
$$

and

$$
\phi_{1}(x) \geq \int_{\Omega} f^{2}(x, y) \mathrm{d} y \int_{\Omega} \phi_{1}(y) \mathrm{d} y, \quad x \in \partial \Omega .
$$

Let $\theta>0$ be large enough such that

$$
2 \theta \min _{\bar{\Omega}} \phi_{1}(x) \geq \max _{\bar{\Omega}}\left|\Delta \phi_{1}\right|+2\left(\max _{\bar{\Omega}} \phi_{1}\right)^{\frac{1+\theta_{1}+\theta_{2}}{2}}+2\left(\max _{\bar{\Omega}} \phi_{1}\right)^{\frac{1+\theta_{3}+\theta_{4}}{2}} .
$$
that

Set $\bar{\omega}(x, t)=\exp (2 \theta t) \phi_{1}(x)$ for $(x, t) \in \Omega \times(0, \infty)$, then it is easy to check

$$
\begin{cases}\bar{\omega}_{t}-\Delta \bar{\omega} \geq 2 \bar{\omega}^{\frac{1+\theta_{1}}{2}} \omega^{\frac{\theta_{2}}{2}}\left(x_{0}, t\right)+2 \bar{\omega}^{\frac{1+\theta_{3}}{2}} \omega^{\frac{\theta_{4}}{2}}\left(x_{0}, t\right), & x \in \Omega, t>0, \\ \bar{\omega}(x, t) \geq \int_{\Omega} f^{2}(x, y) \mathrm{d} y \int_{\Omega} \bar{\omega}(y, t) \mathrm{d} y, & x \in \partial \Omega, t>0, \\ \bar{\omega}(x, 0)>\omega_{0}^{2}(x), & x \in \Omega .\end{cases}
$$

Take $\widetilde{\omega}(x, t)=\bar{\omega}^{\frac{1}{2}}(x, t)$. Then it follows that

$$
\begin{cases}\widetilde{\omega}_{t}-\Delta \widetilde{\omega} \geq \widetilde{\omega}^{\theta_{1}} \widetilde{\omega}^{\theta_{2}}\left(x_{0}, t\right)+\widetilde{\omega}^{\theta_{3}} \widetilde{\omega}^{\theta_{4}}\left(x_{0}, t\right), & x \in \Omega, t>0, \\ \widetilde{\omega}(x, t) \geq \int_{\Omega} f(x, y) \widetilde{\omega}(y, t) \mathrm{d} y, & x \in \partial \Omega, t>0, \\ \widetilde{\omega}(x, 0)>\omega_{0}(x), & x \in \Omega .\end{cases}
$$

This implies that $\widetilde{\omega}$ is a global supersolution of (3.1). The proof is complete. 
Theorem 3.1. If $p q \leq 1$, then all solutions of (1.1), (1.5) are global.

Proof. Since $p q \leq 1$, there exist two constants $\alpha, \beta \in(0,1)$ such that

$$
p \leq \frac{\alpha}{\beta} \leq \frac{1}{q}
$$

Define $k=\frac{1}{\alpha}+\frac{1}{\beta}$. Let $\Phi(x, y) \geq \max \{\varphi(x, y), \psi(x, y)\}$ be a continuous function defined for $(x, y) \in \partial \Omega \times \bar{\Omega}$ and set

$$
a(x)=\left(\int_{\Omega} \Phi(x, y) \mathrm{d} y\right)^{\frac{1-\alpha}{\alpha}}, b(x)=\left(\int_{\Omega} \Phi(x, y) \mathrm{d} y\right)^{\frac{1-\beta}{\beta}}, x \in \partial \Omega .
$$

Suppose $z(x, t)$ solves

$$
\begin{cases}z_{t}-\Delta z=k z^{1-\alpha} z^{p \beta}\left(x_{0}, t\right)+k z^{1-\beta} z^{q \alpha}\left(x_{0}, t\right), & x \in \Omega, t>0, \\ z(x, t)=(a(x)+b(x)+1) \int_{\Omega}\left(\Phi(x, y)+\frac{1}{|\Omega|}\right) z(y, t) \mathrm{d} y, & x \in \partial \Omega, t>0, \\ z(x, 0)=1+u_{0}^{\frac{1}{\alpha}}(x)+v_{0}^{\frac{1}{\beta}}(x), & x \in \Omega .\end{cases}
$$

Notice that (3.2) implies that $1-\alpha+p \beta \leq 1$ and $1-\beta+q \alpha \leq 1$. In view of Lemma 3.1 we know that $z$ is global. Moreover, $z>1$ in $\bar{\Omega} \times[0, \infty)$ by the maximum principle. Set $(\bar{u}, \bar{v})=\left(z^{\alpha}, z^{\beta}\right)$. A simple computation yields

$$
\begin{aligned}
\bar{u}_{t}=\alpha z^{\alpha-1} z_{t} & \geq \alpha z^{\alpha-1}\left(\Delta z+k z^{1-\alpha} z^{p \beta}\left(x_{0}, t\right)\right) \\
& =\alpha z^{\alpha-1} \Delta z+k \alpha z^{p \beta}\left(x_{0}, t\right), \\
\Delta \bar{u}=\alpha z^{\alpha-1} \Delta z & +\alpha(\alpha-1) z^{\alpha-2}|\nabla z|^{2} \leq \alpha z^{\alpha-1} \Delta z,
\end{aligned}
$$

and thus we have

$$
\bar{u}_{t}-\Delta \bar{u} \geq k \alpha z^{p \beta}\left(x_{0}, t\right) \geq\left(z^{\beta}\left(x_{0}, t\right)\right)^{p}=\bar{v}^{p}\left(x_{0}, t\right) .
$$

For $(x, t) \in \partial \Omega \times(0, \infty)$, we have by using Hölder's inequality

$$
\begin{aligned}
\bar{u}(x, t) & \geq(a(x))^{\alpha}\left(\int_{\Omega} \Phi(x, y) z(y, t) \mathrm{d} y\right)^{\alpha} \\
& =\left(\int_{\Omega} \Phi(x, y) \mathrm{d} y\right)^{1-\alpha}\left(\int_{\Omega} \Phi(x, y) z(y, t) \mathrm{d} y\right)^{\alpha} \\
& \geq\left(\int_{\Omega} \varphi(x, y) \mathrm{d} y\right)^{1-\alpha}\left(\int_{\Omega} \varphi(x, y) z(y, t) \mathrm{d} y\right)^{\alpha} \\
& \geq \int_{\Omega} \varphi(x, y) z^{\alpha}(y, t) \mathrm{d} y \\
& =\int_{\Omega} \varphi(x, y) \bar{u}(y, t) \mathrm{d} y .
\end{aligned}
$$

Similarly, we have

$$
\begin{gathered}
\bar{v}_{t}-\Delta \bar{v} \geq \bar{u}^{q}\left(x_{0}, t\right), \quad x \in \Omega, t>0 \\
\bar{v}(x, t) \geq \int_{\Omega} \psi(x, y) \bar{v}(y, t) \mathrm{d} y, \quad x \in \partial \Omega, t>0 .
\end{gathered}
$$


Noticing that $u_{0}(x)<\bar{u}_{0}(x), v_{0}(x)<\bar{v}_{0}(x)$ in $\Omega$, we see that $(\bar{u}, \bar{v})$ is a global supersolution of $(1.1),(1.5)$. Comparison principle implies that $(u, v)<(\bar{u}, \bar{v})$, and hence $(u, v)$ exists globally. This completes the proof.

Theorem 3.2. Suppose that $p q>1$.

(i) For any nonnegative $\varphi(x, y)$ and $\psi(x, y)$, solutions of $(1.1),(1.5)$ blow up in finite time provided that the initial data are large enough.

(ii) If $\int_{\Omega} \varphi(x, y) \mathrm{d} y \geq 1, \int_{\Omega} \psi(x, y) \mathrm{d} y \geq 1$ for any $x \in \partial \Omega$, then any solution to (1.1), (1.5) with positive initial data blows up in finite time.

(iii) If $\int_{\Omega} \varphi(x, y) \mathrm{d} y<1, \int_{\Omega} \psi(x, y) \mathrm{d} y<1$ for any $x \in \partial \Omega$, then solutions to (1.1), (1.5) with small initial data exist globally.

Proof. (i) Let $(\underline{u}, \underline{v})$ be the solution to the homogeneous Dirichlet boundary problem. Then it is well known that for sufficiently large initial data, the solution $(\underline{u}, \underline{v})$ blows up in finite time when $p q>1$ (see [16]). On the other hand, it is obvious that $(\underline{u}, \underline{v})$ is a subsolution of (1.1), (1.5). Hence, the solutions of (1.1), (1.5) with large initial data blow up in finite time provide that $p q>1$.

(ii) Consider the following ODE system

$$
\left\{\begin{array}{l}
f^{\prime}(t)=g^{p}(t), g^{\prime}(t)=f^{q}(t), t>0, \\
f(0)=a>0, g(0)=b>0,
\end{array}\right.
$$

where $a=\frac{1}{2} \min _{\bar{\Omega}} u_{0}(x), b=\frac{1}{2} \min _{\bar{\Omega}} v_{0}(x)$. We know from the theory of ODE that $p q>1$ implies that $(f, g)$ blows up in finite time (see also [29]). Under the assumptions that $\int_{\Omega} \varphi(x, y) \mathrm{d} y \geq 1, \int_{\Omega} \psi(x, y) \mathrm{d} y \geq 1$ for any $x \in \partial \Omega,(f, g)$ is a subsolution of Problems (1.1), (1.5). Therefore, by Proposition 2.1, we see that the solution $(u, v)$ of $(1.1),(1.5)$ satisfies $(u, v)>(f, g)$ and thus $(u, v)$ also blows up in finite time.

(iii) Let $\Psi_{1}(x)$ and $\Psi_{2}(x)$ be the positive solutions of the following linear elliptic problems

$$
-\Delta \Psi_{1}=\epsilon_{0}, \quad x \in \Omega, \quad \Psi_{1}(x)=\int_{\Omega} \varphi(x, y) \mathrm{d} y, \quad x \in \partial \Omega,
$$

and

$$
-\Delta \Psi_{2}=\epsilon_{0}, \quad x \in \Omega, \quad \Psi_{2}(x)=\int_{\Omega} \psi(x, y) \mathrm{d} y, \quad x \in \partial \Omega,
$$

respectively, where $\epsilon_{0}$ is positive constant such that $0 \leq \Psi_{i} \leq 1(i=1,2)$. Such $\epsilon_{0}$ exists since $\int_{\Omega} \varphi(x, y) \mathrm{d} y, \int_{\Omega} \psi(x, y) \mathrm{d} y<1$ for all $x \in \partial \Omega$.

Set

$$
\bar{u}=a \Psi_{1}(x), \bar{v}=b \Psi_{2}(x),
$$

where $a=\epsilon_{0}^{\frac{p+1}{p q-1}}, b=\epsilon_{0}^{\frac{q+1}{p q-1}}$. We shall show that $(\bar{u}, \bar{v})$ is a supersolution of (1.1), (1.5) for small initial data $\left(u_{0}, v_{0}\right)$. Indeed, it follows from $a^{p}=b \epsilon_{0}, b^{q}=a \epsilon_{0}$ that, for $x \in \Omega$,

$$
\bar{u}_{t}-\Delta \bar{u}=a \epsilon_{0}=b^{p} \geq \bar{v}^{p}\left(x_{0}, t\right),
$$




$$
\bar{v}_{t}-\Delta \bar{v}=b \epsilon_{0}=a^{q} \geq \bar{u}^{q}\left(x_{0}, t\right) .
$$

For any $x \in \partial \Omega, t>0$, we have

$$
\begin{aligned}
& \bar{u}(x, t)=a \int_{\Omega} \varphi(x, y) \mathrm{d} y \geq \int_{\Omega} \varphi(x, y) a \Psi_{1}(y) \mathrm{d} y=\int_{\Omega} \varphi(x, y) \bar{u}(y, t) \mathrm{d} y, \\
& \bar{v}(x, t)=b \int_{\Omega} \psi(x, y) \mathrm{d} y \geq \int_{\Omega} \psi(x, y) b \Psi_{2}(y) \mathrm{d} y=\int_{\Omega} \psi(x, y) \bar{v}(y, t) \mathrm{d} y .
\end{aligned}
$$

Here we use $\Psi_{i} \leq 1(i=1,2)$. The above inequalities show that $(\bar{u}, \bar{v})$ is a supersolution of (1.1), (1.5) provide that $u_{0}(x)<a \Psi_{1}(x), v_{0}(x)<b \Psi_{2}(x)$. Therefore, all solutions of (1.1), (1.5) are global in this case. The proof is complete.

Remark 3.1. We would like to remark that Problems (1.2), (1.5) share the same blow-up criteria as Problems (1.1), (1.5). In fact, we can establish a lemma similar to Lemma 3.1 and obtain two theorems similar to Theorems 3.1 and 3.2 for Problems (1.2), (1.5), by applying Lemma 2.2 and Proposition 2.1. See [14] for some similar results.

Remark 3.2. We see from Theorem 3.1 that any weight function on the boundary has no influence on the global existence of solutions when $p q \leq 1$, while Theorem 3.2 shows that they play an substantial role when $p q>1$. In particular, (ii) of Theorem 3.2 is completely different from the case of homogeneous Dirichlet boundary condition.

The following theorem establishes the global existence and blow-up criteria for the exponent-type systems (1.3) and (1.4).

Theorem 3.3. (i) Suppose that $\int_{\Omega} \varphi(x, y) \mathrm{d} y<1, \int_{\Omega} \psi(x, y) \mathrm{d} y<1$ for any $x \in \partial \Omega$. Then for any given initial datum $\left(u_{0}, v_{0}\right)$, the solutions to $(1.3),(1.5)$ and (1.4), (1.5) exist globally when $\lambda$ and $\mu$ are sufficiently small.

(ii) All solutions of (1.3), (1.5) and (1.4), (1.5) with large initial data blow up in finite time.

(iii) Suppose that $\int_{\Omega} \varphi(x, y) \mathrm{d} y \geq 1, \int_{\Omega} \psi(x, y) \mathrm{d} y \geq 1$ for all $x \in \partial \Omega$. Then any solution of (1.3), (1.5) and (1.4), (1.5) with positive initial data blows up in finite time.

Proof. (i) Let $\Psi_{1}(x)$ and $\Psi_{2}(x)$ be defined as in (3.4) and (3.5), respectively, and $(u, v)$ be a solution of $(1.3),(1.5)$. For any given initial datum $\left(u_{0}, v_{0}\right)$, there exist constants $a, b>0$ such that

$$
u_{0}(x)<a \Psi_{1}(x), v_{0}(x)<b \Psi_{2}(x), x \in \bar{\Omega} .
$$

We shall show that $(\bar{u}, \bar{v})=\left(a \Psi_{1}(x), b \Psi_{2}(x)\right)$ is a supersolution of (1.3), (1.5) for small coefficients $\lambda$ and $\mu$. Indeed, if $\lambda \leq \frac{a \epsilon_{0}}{\exp (p b)}, \mu \leq \frac{b \epsilon_{0}}{\exp (a q)}$, we have

$$
\begin{aligned}
& \bar{u}_{t}-\Delta \bar{u}=a \epsilon_{0} \geq \lambda \exp (p b) \geq \lambda \exp \left(p \bar{v}\left(x_{0}, t\right)\right), x \in \Omega, t>0, \\
& \bar{v}_{t}-\Delta \bar{v}=b \epsilon_{0} \geq \mu \exp (a q) \geq \mu \exp \left(q \bar{u}\left(x_{0}, t\right)\right), x \in \Omega, t>0 .
\end{aligned}
$$


For $(x, t) \in \partial \Omega \times(0, \infty)$, it holds

$$
\begin{aligned}
& \bar{u}(x, t)=a \Psi_{1}(x)=a \int_{\Omega} \varphi(x, y) \mathrm{d} y \geq \int_{\Omega} \varphi(x, y) \bar{u}(y, t) \mathrm{d} y, \\
& \bar{v}(x, t)=b \Psi_{2}(x)=b \int_{\Omega} \psi(x, y) \mathrm{d} y \geq \int_{\Omega} \psi(x, y) \bar{v}(y, t) \mathrm{d} y .
\end{aligned}
$$

Thus we obtain by the comparison principle that $(u, v)<(\bar{u}, \bar{v})$, and hence it exists globally.

(ii) Applying the basic inequality $e^{s} \geq \frac{1}{2} s^{2}$ and making use of (i) of Theorem 3.2 , we can easily get the desired result in this case.

(iii) Again by using $e^{s} \geq \frac{1}{2} s^{2}$ and $\int_{\Omega} \varphi(x, y) \mathrm{d} y \geq 1, \int_{\Omega} \psi(x, y) \mathrm{d} y \geq 1$, we see that the solution $(f, g)$ of the following ODE

$$
\left\{\begin{array}{l}
f^{\prime}(t)=\frac{1}{2} \lambda p^{2} g^{2}(t), g^{\prime}(t)=\frac{1}{2} \mu q^{2} f^{2}(t), t>0 \\
f(0)=\frac{1}{2} \min _{\bar{\Omega}} u_{0}(x)>0, g(0)=\frac{1}{2} \min _{\bar{\Omega}} v_{0}(x)>0
\end{array}\right.
$$

is a subsolution of (1.3), (1.5). On the other hand, $(f, g)$ blows up in finite time and so does $(u, v)$.

For Problems (1.4), (1.5), we can prove our conclusion similarly by taking almost the same super and subsolutions and using Proposition 2.1. The proof is completed.

\section{Blow-up profiles}

In this section, we investigate the asymptotic behavior of blow-up solutions near the blow-up time for the case of $\int_{\Omega} \varphi(x, y) \mathrm{d} y \geq 1, \int_{\Omega} \psi(x, y) \mathrm{d} y \geq 1(x \in$ $\partial \Omega$ ). Our main results are the following two theorems on the blow-up profiles of solutions to Problems (1.1)-(1.4).

Theorem 4.1. Suppose that $\left(u_{0}(x), v_{0}(x)\right)>(0,0)$ for $x \in \bar{\Omega}$ and that

$$
\int_{\Omega} \varphi(x, y) \mathrm{d} y \geq 1, \int_{\Omega} \psi(x, y) \mathrm{d} y \geq 1 \text { for any } x \in \partial \Omega .
$$

Let $(u, v)$ be a solution to Problems (1.1), (1.5) or (1.2), (1.5) which blows up at a finite time $T$. Then we have

(i) for the case of localized sources (1.1), (1.5),

$$
\begin{aligned}
& \lim _{t \rightarrow T} u(x, t)(T-t)^{(p+1) /(p q-1)}=\left(\frac{p q-1}{p+1}\left(\frac{p+1}{q+1}\right)^{p /(p+1)}\right)^{-(p+1) /(p q-1)}, \\
& \lim _{t \rightarrow T} v(x, t)(T-t)^{(q+1) /(p q-1)}=\left(\frac{p q-1}{q+1}\left(\frac{q+1}{p+1}\right)^{q /(q+1)}\right)^{-(q+1) /(p q-1)},
\end{aligned}
$$

uniformly on $\bar{\Omega}$;

(ii) for the case of nonlocal sources (1.2), (1.5),

$$
\lim _{t \rightarrow T} u(x, t)(T-t)^{(p+1) /(p q-1)}=\left(|\Omega| \frac{p q-1}{p+1}\left(\frac{p+1}{q+1}\right)^{p /(p+1)}\right)^{-(p+1) /(p q-1)},
$$




$$
\lim _{t \rightarrow T} v(x, t)(T-t)^{(q+1) /(p q-1)}=\left(|\Omega| \frac{p q-1}{q+1}\left(\frac{q+1}{p+1}\right)^{q /(q+1)}\right)^{-(q+1) /(p q-1)},
$$

uniformly on $\bar{\Omega}$.

Theorem 4.2. Suppose that $\left(u_{0}(x), v_{0}(x)\right)>(0,0)$ for $x \in \bar{\Omega}$ and that

$$
\int_{\Omega} \varphi(x, y) \mathrm{d} y \geq 1, \int_{\Omega} \psi(x, y) \mathrm{d} y \geq 1 \text { for any } x \in \partial \Omega .
$$

Let $(u, v)$ be a solution to Problems (1.3), (1.5) or (1.4), (1.5) which blows up at a finite time $T$. Then there exists a constant $C>0$ such that

$$
\begin{aligned}
& \ln (T-t)^{-\frac{1}{q}}-C \leq u(x, t) \leq \ln (T-t)^{-\frac{1}{q}}+C, \quad(x, t) \in \bar{\Omega} \times(0, T), \\
& \ln (T-t)^{-\frac{1}{p}}-C \leq v(x, t) \leq \ln (T-t)^{-\frac{1}{p}}+C, \quad(x, t) \in \bar{\Omega} \times(0, T) .
\end{aligned}
$$

Corollary 4.1. Assume that all the conditions in Theorem 4.2 hold. Then, we have

$$
\lim _{t \rightarrow T}|\ln (T-t)|^{-1} u(x, t)=\frac{1}{q}, \lim _{t \rightarrow T}|\ln (T-t)|^{-1} v(x, t)=\frac{1}{p},
$$

uniformly on $\bar{\Omega}$.

Remark 4.1. Theorems 4.1 and 4.2 show that the blow-up set of a blow-up solution to Problems (1.1)-(1.4) with (1.5) is the whole domain $\bar{\Omega}$.

Since the systems (1.1)-(1.4) are completely coupled, we have $\lim _{t \rightarrow T}\|u\|_{\infty}=$ $+\infty$ and $\lim _{t \rightarrow T}\|v\|_{\infty}=+\infty$ provided that the solution $(u, v)$ blows up in a finite time $T$ (see Lemma 4.1). We first establish a lemma which will play an essential role in what follows. For convenience, we rewrite our systems into a general form

$$
\begin{cases}u_{t}-\Delta u=g_{1}(t), v_{t}-\Delta v=g_{2}(t), & (x, t) \in Q_{T}, \\ u(x, t)=\int_{\Omega} \varphi(x, y) u(y, t) d y, & (x, t) \in S_{T}, \\ v(x, t)=\int_{\Omega} \psi(x, y) v(y, t) d y, & (x, t) \in S_{T}, \\ u(x, 0)=u_{0}(x), v(x, 0)=v_{0}(x), & x \in \Omega,\end{cases}
$$

where $g_{i}(t)(i=1,2)$ represent the localized or nonlocal sources in (1.1)-(1.4).

Lemma 4.1. Suppose that $\left(u_{0}(x), v_{0}(x)\right)>(0,0)$ for $x \in \bar{\Omega}$ and that

$$
\int_{\Omega} \varphi(x, y) \mathrm{d} y \geq 1, \int_{\Omega} \psi(x, y) \mathrm{d} y \geq 1 \text { for any } x \in \partial \Omega .
$$

Let $(u, v)$ be a solution of Problem (4.1) which blows up at a finite time $T$. Then we have, for all $x \in \bar{\Omega}$ and $t>0$,

$$
G_{1}(t) \leq u(x, t) \leq\left\|u_{0}\right\|_{\infty}+G_{1}(t), G_{2}(t) \leq v(x, t) \leq\left\|v_{0}\right\|_{\infty}+G_{2}(t),
$$

where $G_{i}(t)=\int_{0}^{t} g_{i}(s) \mathrm{d} s(i=1,2)$. 
Proof. Assume that $(u, v)$ blows up in a finite time $T$, i.e., $\lim _{t \rightarrow T}\left(\|u\|_{\infty}+\right.$ $\left.\|v\|_{\infty}\right)=+\infty$. Without loss of generality, we may assume that $\lim _{t \rightarrow T}\|u\|_{\infty}=$ $+\infty$. Let

$$
U(t)=\max _{x \in \bar{\Omega}} u(x, t), V(t)=\max _{x \in \bar{\Omega}} v(x, t) .
$$

Then $U(t)$ and $V(t)$ are Lipschitz continuous and thus are differentiable almost everywhere (see [8]). Moreover, we have from (4.1)

$$
U^{\prime}(t) \leq g_{1}(t), \quad V^{\prime}(t) \leq g_{2}(t) \text { a.e. } t \in[0, T) .
$$

Integrating the two inequalities above from 0 to $t$, one obtains

$$
U(t)-U(0) \leq G_{1}(t), V(t)-V(0) \leq G_{2}(t) .
$$

Thus we see from the first inequality that $\lim _{t \rightarrow T} G_{1}(t)=\infty$, which implies $\limsup _{t \rightarrow T} g_{1}(t)=\infty$. By the relationship between $g_{1}(t)$ and $v(x, t)$ we see that $\lim \sup _{t \rightarrow T}\|v\|_{\infty}=+\infty$. Hence, for the blow-up solution of (4.1), simultaneous blow-up occurs.

Set $J_{1}(x, t)=u(x, t)-G_{1}(t), J_{2}(x, t)=v(x, t)-G_{2}(t)$, then we have

$$
\begin{aligned}
& J_{1 t}(x, t)-\Delta J_{1}=u_{t}-g_{1}(t)-\Delta u=0,(x, t) \in \Omega \times(0, T), \\
& J_{2 t}(x, t)-\Delta J_{2}=v_{t}-g_{2}(t)-\Delta v=0,(x, t) \in \Omega \times(0, T) .
\end{aligned}
$$

For $(x, t) \in \partial \Omega \times(0, T)$, it is clear that

$$
\begin{aligned}
& J_{1}(x, t)=\int_{\Omega} \varphi(x, y) u(y, t) \mathrm{d} y-G_{1}(t) \geq \int_{\Omega} \varphi(x, y) J_{1}(y, t) \mathrm{d} y, \\
& J_{2}(x, t)=\int_{\Omega} \psi(x, y) v(y, t) \mathrm{d} y-G_{2}(t) \geq \int_{\Omega} \psi(x, y) J_{2}(y, t) \mathrm{d} y,
\end{aligned}
$$

where we have used the assumptions that $\int_{\Omega} \varphi(x, y) \mathrm{d} y \geq 1, \int_{\Omega} \psi(x, y) \mathrm{d} y \geq 1$ for all $x \in \partial \Omega$. Obviously, $J_{1}(x, 0)=u_{0}(x)>0, J_{2}(x, 0)=v_{0}(x)>0$. By maximum principle (see Theorem 2.1 in [11]) we have $J_{1}(x, t) \geq 0$ and $J_{2}(x, t) \geq$ 0 for $(x, t) \in \bar{\Omega} \times[0, T)$. This together with (4.3) implies our conclusion. The proof is complete.

Now we are in the position to prove the blow-up profile of $(u, v)$. We usually use the notation $\omega(t) \sim s(t), t \rightarrow T$, if $\lim _{t \rightarrow T} \frac{\omega(t)}{s(t)}=1$. Then we see from (4.2) that

$$
u(x, t) \sim G_{1}(t), t \rightarrow T \text { and } v(x, t) \sim G_{2}(t), t \rightarrow T .
$$

Proof of Theorem 4.1. (i) Denote

$$
g_{1}(t)=v^{p}\left(x_{0}, t\right), g_{2}(t)=u^{q}\left(x_{0}, t\right), G_{i}(t)=\int_{0}^{t} g_{i}(s) \mathrm{d} s(i=1,2) .
$$

From (4.4) we see that

$$
G_{1}^{\prime}(t)=g_{1}(t)=v^{p}\left(x_{0}, t\right) \sim G_{2}^{p}(t), G_{2}^{\prime}(t)=g_{2}(t)=u^{q}\left(x_{0}, t\right) \sim G_{1}^{q}(t), t \rightarrow T .
$$


It follows that

$$
\frac{1}{q+1} G_{1}^{q+1}(t) \sim \frac{1}{p+1} G_{2}^{p+1}(t), \quad t \rightarrow T .
$$

Thus, we easily deduce by (4.5) and (4.6) that

which implies

$$
\begin{aligned}
& G_{1}^{\prime}(t) \sim\left(\frac{p+1}{q+1}\right)^{\frac{p}{p+1}} G_{1}^{\frac{p(q+1)}{p+1}}, t \rightarrow T, \\
& G_{2}^{\prime}(t) \sim\left(\frac{q+1}{p+1}\right)^{\frac{q}{q+1}} G_{2}^{\frac{q(p+1)}{q+1}}, t \rightarrow T,
\end{aligned}
$$

$$
\begin{gathered}
\left(G_{1}^{\frac{1-p q}{p+1}}(t)\right)^{\prime} \sim-\frac{p q-1}{p+1}\left(\frac{p+1}{q+1}\right)^{\frac{p}{p+1}}, t \rightarrow T, \\
\left(G_{2}^{\frac{1-p q}{q+1}}(t)\right)^{\prime} \sim-\frac{p q-1}{q+1}\left(\frac{q+1}{p+1}\right)^{\frac{q}{q+1}}, t \rightarrow T .
\end{gathered}
$$

Integrating the above equivalents form $t$ to $T$, we obtain

$$
\begin{aligned}
& G_{1}(t) \sim\left[\frac{p q-1}{p+1}\left(\frac{p+1}{q+1}\right)^{\frac{p}{p+1}}(T-t)\right]^{-\frac{p+1}{p q-1}}, t \rightarrow T, \\
& G_{2}(t) \sim\left[\frac{p q-1}{q+1}\left(\frac{q+1}{p+1}\right)^{\frac{q}{q+1}}(T-t)\right]^{-\frac{q+1}{p q-1}}, t \rightarrow T .
\end{aligned}
$$

Thus, by Lemma 4.1, we get our conclusion.

(ii) In this case, we take

$$
g_{1}(t)=\int_{\Omega} v^{p}(y, t) \mathrm{d} y, g_{2}(t)=\int_{\Omega} u^{q}(y, t) \mathrm{d} y, G_{i}(t)=\int_{0}^{t} g_{i}(s) \mathrm{d} s(i=1,2) .
$$

Combining the above equalities with (4.4) and using Lebesgue's dominated convergence theorem, one gets

$$
\begin{aligned}
& G_{1}^{\prime}(t)=g_{1}(t)=\int_{\Omega} v^{p}(y, t) \mathrm{d} y \sim|\Omega| G_{2}^{p}(t), t \rightarrow T, \\
& G_{2}^{\prime}(t)=g_{2}(t)=\int_{\Omega} u^{q}(y, t) \mathrm{d} y \sim|\Omega| G_{1}^{q}(t), t \rightarrow T .
\end{aligned}
$$

The remaining arguments are similar to (i), and the details are omitted here. This completes the proof.

At the end of this section, we derive the blow-up rate estimates for the exponent-type systems (1.3), (1.5) and (1.4), (1.5). But we need to be a little more careful in this case, since exponentiation of equivalents is not permitted. In the following arguments, we shall use $c$ and $C$ to denote various generic constants if they cause no confusion. 
Proof of Theorem 4.2. For Problems (1.3), (1.5), we denote

$$
g_{1}(t)=\lambda e^{p v\left(x_{0}, t\right)}, g_{2}(t)=\mu e^{q u\left(x_{0}, t\right)}, G_{i}(t)=\int_{0}^{t} g_{i}(s) \mathrm{d} s(i=1,2), t \in(0, T) .
$$

It follows from Lemma 4.1 and the above equalities that

$$
\left\{\begin{array}{l}
\lambda e^{p G_{2}(t)} \leq G_{1}^{\prime}(t)=g_{1}(t)=\lambda e^{p v\left(x_{0}, t\right)} \leq \lambda e^{p G_{2}(t)+p\left\|v_{0}\right\|_{\infty}} \leq C e^{p G_{2}(t)}, t \in(0, T), \\
\mu e^{q G_{1}(t)} \leq G_{2}^{\prime}(t)=g_{2}(t)=\mu e^{q u\left(x_{0}, t\right)} \leq \mu e^{q G_{1}(t)+q\left\|u_{0}\right\|_{\infty}} \leq C e^{q G_{1}(t)}, t \in(0, T) .
\end{array}\right.
$$

Then, we have from $(4.7)$

$$
c \frac{e^{p G_{2}(t)}}{e^{q G_{1}(t)}} \leq \frac{\mathrm{d} G_{1}(t)}{\mathrm{d} G_{2}(t)} \leq C \frac{e^{p G_{2}(t)}}{e^{q G_{1}(t)}}, \quad t \in(0, T),
$$

which implies

$$
c e^{p G_{2}(t)} \mathrm{d} G_{2} \leq e^{q G_{1}(t)} \mathrm{d} G_{1} \leq C e^{p G_{2}(t)} \mathrm{d} G_{2}, \quad t \in(0, T) .
$$

Integrating the above inequality from $t$ to $T$, we get

$$
p G_{2}(t)-C \leq q G_{1}(t) \leq p G_{2}(t)+C, \quad t \in(0, T) .
$$

Combining this inequality with (4.7), we have

$$
\begin{aligned}
& c e^{q G_{1}(t)} \leq G_{1}^{\prime}(t) \leq C e^{q G_{1}(t)}, \quad t \in(0, T), \\
& c e^{p G_{2}(t)} \leq G_{2}^{\prime}(t) \leq C e^{p G_{2}(t)}, \quad t \in(0, T) .
\end{aligned}
$$

Thus, it can be deduced by integrating the above inequalities over $(t, T)$ that

$$
\begin{aligned}
& \ln (T-t)-C \leq-q G_{1}(t) \leq \ln (T-t)+C, \quad t \in(0, T), \\
& \ln (T-t)-C \leq-p G_{2}(t) \leq \ln (T-t)+C, \quad t \in(0, T),
\end{aligned}
$$

which, together with Lemma 4.1, implies that

$$
\begin{aligned}
& -\ln (T-t)^{\frac{1}{q}}-C \leq u(x, t) \leq-\ln (T-t)^{\frac{1}{q}}+C, \quad(x, t) \in \Omega \times(0, T), \\
& -\ln (T-t)^{\frac{1}{p}}-C \leq v(x, t) \leq-\ln (T-t)^{\frac{1}{p}}+C, \quad(x, t) \in \Omega \times(0, T) .
\end{aligned}
$$

For Problems (1.4), (1.5), we take

$$
\begin{gathered}
g_{1}(t)=\lambda \int_{\Omega} e^{p v(y, t)} \mathrm{d} y, g_{2}(t)=\mu \int_{\Omega} e^{q u(y, t)} \mathrm{d} y, \\
G_{i}(t)=\int_{0}^{t} g_{i}(s) \mathrm{d} s, \quad(i=1,2), \quad t \in(0, T) .
\end{gathered}
$$

By using similar arguments as (1.3), (1.5), we can prove our results. The proof of Theorem 4.2 is complete. 
Remark 4.2. Results similar to Theorems 3.1, 3.2 and 4.1 also hold for the following system $u_{t}=\Delta u+\|v\|_{p}^{l}, v_{t}=\Delta v+\|u\|_{q}^{s}$ coupled with the initial and boundary conditions (1.5), where $p, q \geq 1, l, s>0$ and $\|\cdot\|_{r} \equiv\left(\int_{\Omega}|\cdot|^{r} d x\right)^{1 / r}$. More precisely, by establishing a lemma similar to Lemma 3.1, one can prove that all the conclusions of Theorems 3.1 and 3.2 are still valid for this problem if the conditions $p q \leq 1$ and $p q>1$ are replaced by $l s \leq 1$ and $l s>1$, respectively. As for the uniform blow-up profile, (ii) of Theorem 4.1 also holds for such a problem, only with some different constants on the right hand side of the equalities in Theorem 4.1.

Remark 4.3. We would like to remark that many authors have studied the blow-up rate or blow-up profile of parabolic equations or systems with nonlocal boundary conditions when $\int_{\Omega} \varphi(x, y) \mathrm{d} y \leq 1, \int_{\Omega} \psi(x, y) \mathrm{d} y \leq 1, x \in \partial \Omega$. See $[14,26,28]$ for example. However, the case $\int_{\Omega} \varphi(x, y) \mathrm{d} y \geq 1, \int_{\Omega} \psi(x, y) \mathrm{d} y \geq$ $1, x \in \partial \Omega$ is seldom studied. Theorems 4.1 and 4.2 show that the blow-up solutions have uniform blow-up profile and the blow-up rate estimates hold uniformly on $\bar{\Omega}$, which is different from the previous results mentioned above.

Acknowledgment. The authors would like to thank the referees for their valuable comments on and suggestions regarding the original manuscript.

\section{References}

[1] J. R. Anderson and K. Deng, Global existence for degenerate parabolic equations with a non-local forcing, Math. Methods Appl. Sci. 20 (1997), no. 13, 1069-1087.

[2] J. Bebernes and D. Eberly, Mathematical Problems from Combustion Theory, SpringerVerlag, New York, 1989.

[3] K. Bimpong-Bota, P. Ortoleva, and J. Ross, Far-from-equilibrium phenomena at local cites of reaction, J. Chem. Phys. 60 (1974), 3124-3133.

[4] D. E. Carlson, Linear thermoelasticity, Encyclopedia, vol. vIa/2, Springer, Berlin, 1972.

[5] J. M. Chadam, A. Peirce, and H. M. Yin, The blowup property of solutions to some diffusion equations with localized nonlinear reactions, J. Math. Anal. Appl. 169 (1992), no. 2 313-328.

[6] Y. Chen and H. Gao, Asymptotic blow-up behavior for a nonlocal degenerate parabolic equation, J. Math. Anal. Appl. 330 (2007), no. 2, 852-863.

[7] A. Friedman, Monotonic decay of solutions of parabolic equations with nonlocal boundary conditions, Quart. Appl. Math. 44 (1986), no. 3, 401-407.

[8] A. Friedman and J. B. Mcleod, Blow-up of positive solutions of semilinear heat equations, Indiana Univ. Math. J. 34 (1985), no. 2, 425-447.

[9] W. A. Day, Extensions of a property of the heat equation to linear thermoelasticity and other theories, Quart. Appl. Math. 40 (1982), no. 3, 319-330.

[10] _ A decreasing property of solutions of parabolic equations with applications to thermoelasticity, Quart. Appl. Math. 40 (1983), no. 4, 468-475.

[11] K. Deng, Comparison principle for some nonlocal problems, Quart. Appl. Math. 50 (1992), no. 3, 517-522.

[12] K. Deng and H. A. Levine, The role of critical exponents in blow-up theorems: the sequel, J. Math. Anal. Appl. 243 (2000), no. 1, 85-126.

[13] Y. Han and W. Gao, Global existence and blow-up for a class of degenerate parabolic systems with localized source, Acta Appl. Math. 112 (2010), no. 2, 251-261. 
[14] L. H. Kong and M. X. Wang, Global existence and blow-up of solutions to a parabolic system with nonlocal sources and boundaries, Sci. China Ser. A 50 (2007), no. 9, 12511266.

[15] H. A. Levine, The role of critical exponents in blow up theorems, SIAM Rev. 32 (1990), 262-288.

[16] H. L. Li and M. X. Wang, Properties of blow-up solutions to a parabolic system with nonlinear localized terms, Discrete Contin. Dyn. Syst. 13 (2005), no. 3, 683-700.

[17] P. Ortoleva and J. Ross, Local structures in chemical reactions with heterogeneous catalysis, J. Chem. Phys. 56 (1972), 4397-4400.

[18] C. V. Pao, Nonlinear Parabolic and Elliptic Equations, Plenum, New York, 1992.

[19] _ Dynamics of reaction-diffusion equations with nonlocal boundary conditions, Quart. Appl. Math. 50 (1995), no. 1, 173-186.

[20] - Asymptotic behavior of solutions of reaction-diffusion equations with nonlocal boundary conditions, J. Comput. Appl. Math. 88 (1998), no. 1, 225-238.

[21] N Numerical solutions of reaction-diffusion equations with nonlocal boundary conditions, J. Comput. Appl. Math. 136 (2001), no. 1-2, 227-243.

[22] S. Seo, Blowup of solutions to heat equations with nonlocal boundary conditions, Kobe J. Math. 13 (1996), no. 2, 123-132.

[23] Global existence and decreasing property of boundary values of solutions to parabolic equations with nonlocal boundary conditions, Pacific J. Math. 193 (2000), no. 1, 219-226.

[24] P. Souplet, Blow up in nonlocal reaction-diffusion equations, SIAM J. Math. Anal. 29 (1998), no. 6, 1301-1334.

[25] _ Uniform blow-up profiles and boundary behavior for diffusion equations with nonlocal nonlinear source, J. Differential Equations 153 (1999), no. 2, 374-406.

[26] Y. L. Wang, C. L. Mu, and Z. Y. Xiang, Blowup of solutions to a porous medium equation with nonlocal boundary condition, Appl. Math. Comput. 192 (2007), no. 2, 579-585.

[27] M. X. Wang and Y. M. Wang, Properties of positive solutions for non-local reactiondiffusion problems, Math. Methods Appl. Sci. 19 (1996), no. 14, 1141-1156.

[28] Y. L. Wang and Z. Y. Xiang, Blowup analysis for a semilinear parabolic system with nonlocal boundary condition, Boundary Value Problems 2009 (2009), Article ID 516390, 14 pages.

[29] Z. Y. Xiang, X. G. Hu, and C. L. Mu, Neumann problem for reaction-diffusion systems with nonlocal nonlinear sources, Nonlinear Anal. 61 (2005), no. 7, 1209-1224.

[30] H. M. Yin, On a class of parabolic equations with nonlocal boundary conditions, J. Math. Anal. Appl. 294 (2004), no. 2, 712-728.

[31] Y. F. Yin, On nonlinear parabolic equations with nonloal boundary conditions, J. Math. Anal. Appl. 185 (1994), no. 1, 161-174.

[32] S. N. Zheng and L. H. Kong, Roles of weight functions in a nonlinear nonlocal parabolic system, Nonlinear Anal. 68 (2008), no. 8, 2406-2416.

YUZHU HAN

InSTITUTE OF Mathematics

JILIN UNIVERSITY

Changchun 130012, P. R. China

E-mail address: hanyuzhu2003@yahoo.cn

Wenjie Gao

Institute of MatheMATICS

JILIN UNIVERSITY

Changchun 130012, P. R. China

E-mail address: wjgao@jlu.edu.cn 